\title{
PLANETESIMALS IN THE PRESENCE OF GIANT PLANET MIGRATION
}

\author{
Graeme Lufkin, Derek C. Richardson, and Lee G. Mundy \\ Department of Astronomy, University of Maryland, College Park, MD 20740-2421; \\ gwl@astro.umd.edu,dcr@astro.umd.edu, lgm@astro.umd.edu \\ Received 2006 March 22; accepted 2006 August 16
}

\begin{abstract}
We present $N$-body simulations illustrating how giant planet migration can significantly affect the conditions for the formation of interior terrestrial planets. A giant planet migrating through a swarm of planetesimals will scatter many of them onto very eccentric and inclined orbits, preventing them from playing any further role in additional planet formation. The magnitude of this excitation is strongly dependent on the mass and migration rate of the giant planet. We found that if planet migration occurs for low-mass planets (one-tenth of the mass of Jupiter) or proceeds very rapidly $\left(10^{-3} \mathrm{AU} \mathrm{yr}^{-1}\right)$, planetesimal swarms can dynamically cool via gas drag, collisions, and dynamical friction after the giant passes through. Other simulations produce a population of planetesimals on orbits with very high eccentricity and inclination, which may persist and become a signature of a previous migration event. In some cases further growth of terrestrial planets is possible, leading to our prediction of terrestrial planets existing on orbits outside of some hot Jupiters.
\end{abstract}

Subject headings: accretion, accretion disks - methods: $n$-body simulations — planetary systems planets and satellites: general

Online material: color figures

\section{INTRODUCTION}

Planet migration has become an integral part of explaining the observations of extrasolar planets. At present, neither the rate nor the prevalence of such migration is well understood. In almost all scenarios, however, inward migration is rapid for planets more massive than $1 M_{\oplus}$. This migration has obvious consequences for the fate of the planet. Migration also has the potential to strongly affect the characteristics of the disk. In this paper we accept that giant planet migration occurs and investigate its effect on planetesimals initially interior to the planet.

Models of terrestrial planet formation (Chambers 2001), evidence from our solar system (Yin et al. 2002; Jacobsen \& Yin 2003), and models of giant planet formation (Pollack et al. 1996; Mayer et al. 2004) suggest that terrestrial and giant planets may be forming and migrating at the same time. The lure of Earth-like extrasolar planets has motivated many previous studies of terrestrial planet formation in the presence of migrating giant planets. Armitage (2003) assumed that a migrating giant planet completely removes all solid material and showed that the subsequent replenishment time is long, reducing the likelihood of additional planet formation. Subsequent studies have suggested that this assumption may be overly pessimistic. Mandell \& Sigurdsson (2003) found that if migration occurs after terrestrial planets have fully formed (i.e., masses like the current inner solar system), a significant fraction of the planets survive. Recently, Fogg \& Nelson (2005) used $N$-body simulations with multiple levels of physics to show that the effect of giant planet migration on interior solid bodies can be quite complex, for example, enhancing planetesimal growth in sweeping resonances. The method used in this paper is similar to that of Edgar \& Artymowicz (2004), which focused on very rapid migration of planets less massive than Jupiter, as expected from type III migration (Masset \& Papaloizou 2003). This paper presents a parameter search over a wide range of the giant planet mass and migration rate, with the goal of estimating the survivability of solid material initially interior to the giant.

\section{INITIAL CONDITIONS}

We construct a disk of planetesimals as described by Kokubo \& Ida (2002) with Rayleigh distributions for the eccentricity and inclination. For the eccentricity distribution, the mean value was 0.01 ; for the inclination distribution, 0.005 radian. The disk extended from 0.5 to $4.5 \mathrm{AU}$, with a surface number density proportional to $r^{-3 / 2}$. The other three orbital elements (longitude of perihelion, longitude of ascending node, and mean anomaly) were distributed uniformly. This initial disk is in quasi-equilibrium in the sense that the velocity dispersion is comparable to the escape speed from $100 \mathrm{~km}$ size objects. The top left panel of Figure 1 shows the described configuration.

In this investigation we are interested in planetesimals that are large enough to have decoupled from the gas disk, but not so massive that gravitational focusing leads them to undergo oligarchic growth. As such, we will treat the planetesimals as massless, feeling only the gravitational force of the central star and the migrating planet. Although interaction with a gas disk is not explicitly modeled, we discuss the possible effects of gas drag in $\S 4.3$.

\section{NUMERICAL TECHNIQUE AND SETUP}

We wish to follow the trajectories of test particles in the presence of two massive bodies whose configuration changes uniformly and slowly. We used the program PKDGRAV (Richardson et al. 2000; also see Stadel 2001) to calculate gravity and integrate orbits in the disk. Since the planetesimals are treated as test particles, the calculation time is directly proportional to the number of particles. PKDGRAV uses a second-order leapfrog integrator with a binary hierarchy of time steps. The time step for each particle was chosen as a small fraction $(<1 / 600)$ of the orbital period about the dominant perturber, either the planet or the central star. We verified the appropriateness of the integrator by decreasing the time step and finding qualitative convergence in the final distribution of orbital elements and number of ejected particles.

The giant planet is kept on a circular orbit, initially at 5.2 AU. It is migrated inward at a constant rate, denoted $\alpha$, and its mass $\left(M_{p}\right)$ 

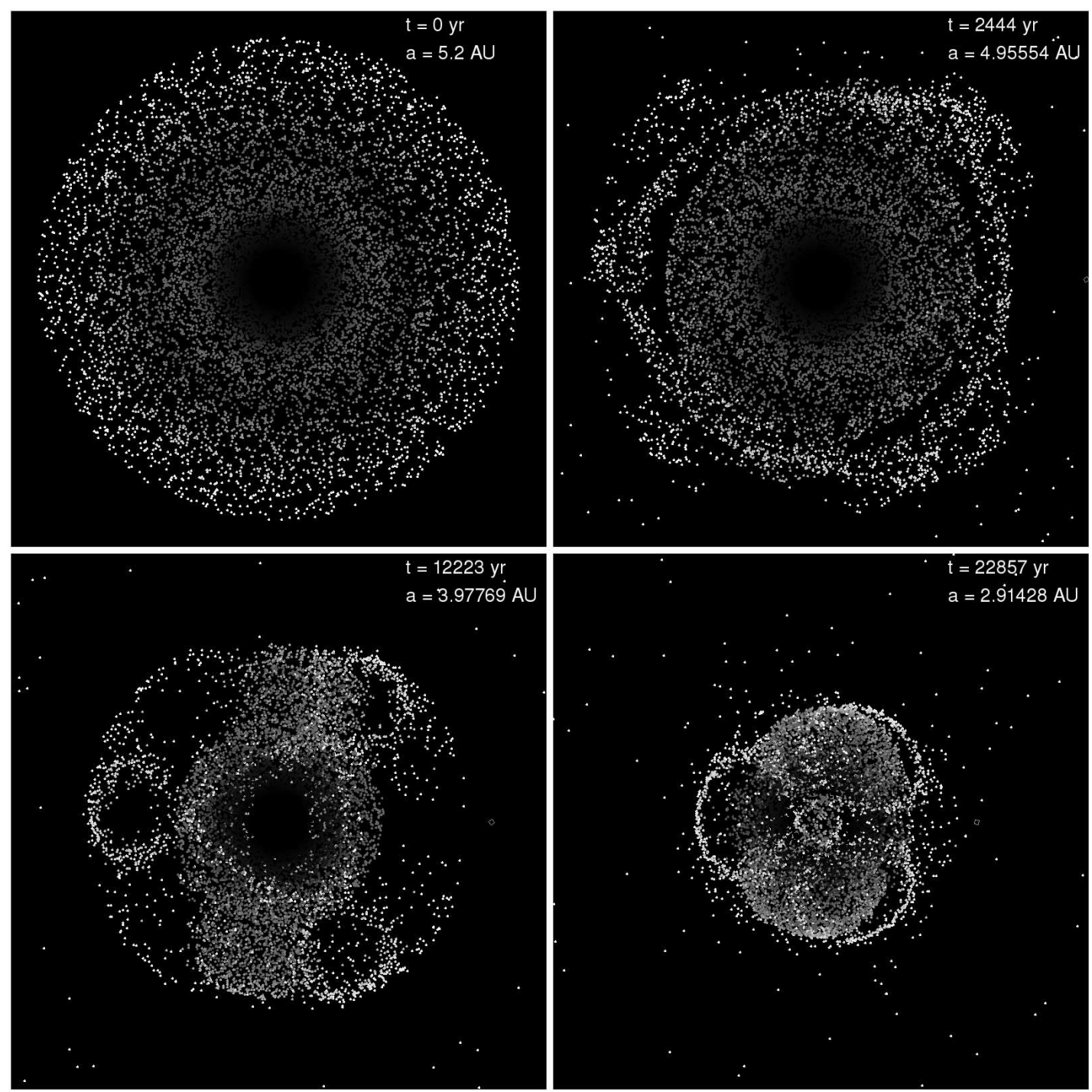

FIG. 1.- Snapshots of a disk of planetesimals under the gravitational influence of a migrating giant planet. The planetesimals are colored by the semimajor axis of their initial orbit. This figure looks down on the disk in a frame that rotates with the giant planet, and is $10 \mathrm{AU}$ in both directions. The simulation time and current semimajor axis of the orbit of the giant planet are shown in the upper right of each frame. Top left: The initial conditions; planetesimals are on nearly circular, nearly coplanar orbits from 0.5 to $4.5 \mathrm{AU}$ from the central star of mass $1 M_{\odot}$. Top right: The giant planet (open diamond, at right edge, centered vertically) enters the frame at the right. As the giant migrates, it carves planetesimals off the disk via resonant interaction. Bottom left: Two- and three-lobed resonant structure is obvious. Bottom right: As the giant planet continues toward the central star, the resonant structure is erased as planetesimals undergo stochastic close encounters. [See the electronic edition of the Journal for a color version of this figure.]

is kept fixed. The simulation is run until the planet comes within $0.01 \mathrm{AU}$ of the central star. This end distance is consistent with the planet accreting onto the star or the migration halting, resulting in a hot Jupiter. Since the planetesimals are massless, no additional dynamical evolution of their orbits occurs after this point.

\section{RESULTS AND DISCUSSION}

The simulation outputs the position and velocity of the planetesimals at regular intervals. From these snapshots we calculate the instantaneous orbital elements. Figure 2 shows the time evolution of orbital elements of a sample of planetesimals from one simulation. Most of the following discussion uses histograms and averages of these elements to quantify the dynamical heating of the disk.

\subsection{Exciting the Disk}

The giant planet immediately starts exciting the planetesimals in the simulation. The initial condition is out of equilibrium be- cause the planet is introduced suddenly, but this affects only the outermost planetesimals. Many particles are captured by resonances and are swept inward as the planet migrates, their orbits simultaneously shrinking and becoming more eccentric. As the eccentricity of a particle increases, it will undergo a close encounter with the planet, drastically changing its orbital elements. Some particles are actually ejected from the system, and many more are pushed to orbits with eccentricity very close to 1 , known as plunging orbits. Particles initially further in are more tightly bound and are not affected until the giant and its low-order resonances migrate nearer. See the progression of snapshots of a system in Figure 1.

\subsection{Dependence on Planet Mass and Migration Rate}

We ran a suite of simulations, independently varying the mass of the migrating planet and the migration rate. For a planet mass of $1 M_{\text {Jup }}$ we varied the migration rate from $10^{-3}$ to $10^{-5} \mathrm{AU} \mathrm{yr}^{-1}$ (high end from simulations of migration due to density wave 

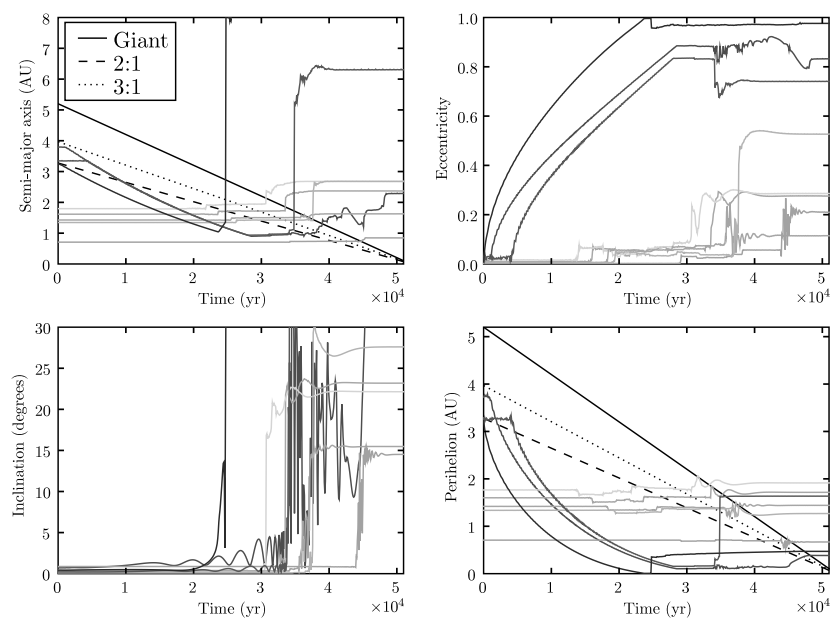

FIG. 2.-Orbital element history of planetesimals in the presence of a migrating giant planet. The paths shown are for eight planetesimals randomly chosen from the simulation. Note how some of the particles get pushed inward ahead of the planet, their orbits simultaneously elongating and shrinking. For the semimajor axis and perihelion plots (top left, bottom right), the path of the migrating giant planet (solid diagonal line) and its two strongest resonances (dashed and dotted lines) are shown. [See the electronic edition of the Journal for a color version of this figure.]

torques, Nelson \& Benz [2003]; low end from estimates of viscous migration within a gap, Ward [1997]); for a migration rate of $10^{-4} \mathrm{AU} \mathrm{yr}^{-1}$ we varied the planet mass from $0.1 M_{\text {Jup }}$ to $2.0 M_{\text {Jup }}$. Figure 3 shows histograms of the final distribution of orbital elements for certain values of the planet mass and migration rate. The mean, median, and variance of the final eccentricity and inclination of the planetesimals are shown in Figure 4 as functions of the planet mass and migration rate. Lower-mass perturbing planets have weaker resonances, causing less excitation of the swarm. Similarly, a faster migration rate means that many particles will miss being caught in resonance. For either of these
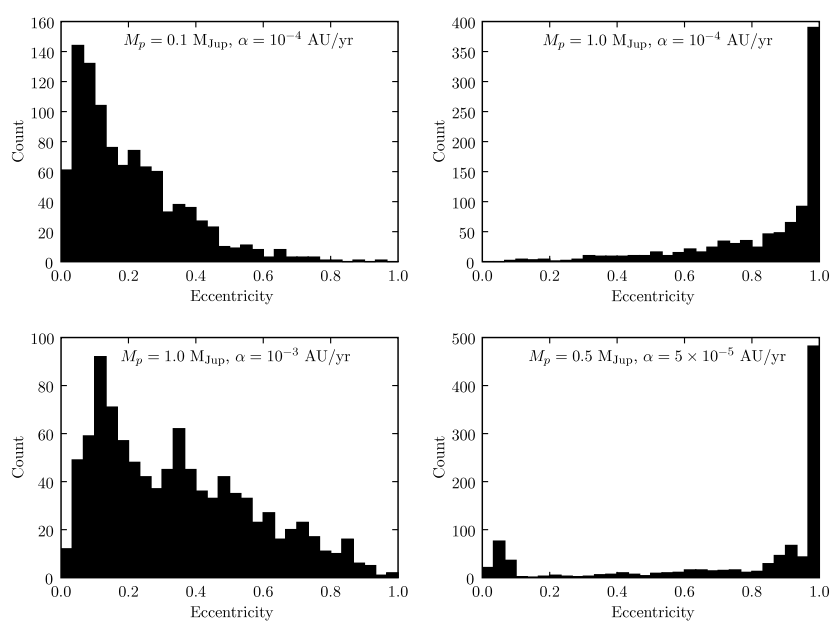

FIG. 3.-Histograms of final eccentricity showing influence of planet mass and migration rate. Top left: A low-mass giant planet migrating at our "average" rate increases the eccentricity of most planetesimals, but does not saturate the excitation. Top right: A more massive planet migrating at the same rate ejected $15 \%$ of the swarm and pushed most of the other planetesimals onto very high eccentricity, or plunging, orbits. Bottom left: A massive planet migrating 10 times faster leaves a broad spread of eccentricities. Bottom right: A low-mass planet can still completely excite the disk if it migrates slower, allowing more time for secular interactions.
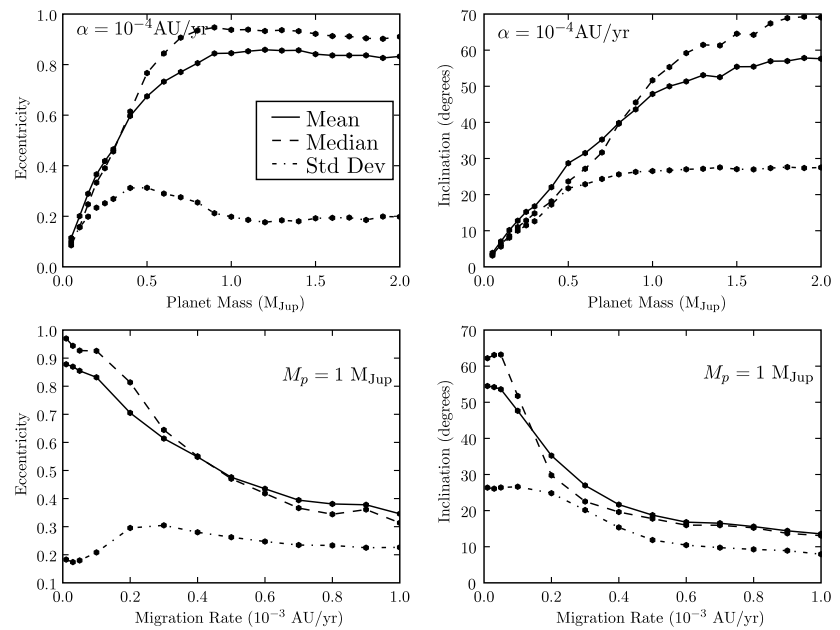

FIG. 4.-Dependence of eccentricity and inclination on planet mass and migration rate. Top panels: We held the giant planet migration rate fixed at $\alpha=10^{-4} \mathrm{AU} \mathrm{yr}^{-1}$ and varied the mass of the planet; shown are the final mean, median, and standard deviation of the eccentricity (left) and inclination (right) of the planetesimals. Bottom panels: We exchange the varied parameters, holding the giant planet mass fixed at $1 M_{\mathrm{Jup}}$ and varying the migration rate. The dependence on both parameters is strong. We show only bound planetesimals, so the eccentricity is limited to be $<1$. While the mean and median value of eccentricity of bound planetesimals plateaus at high mass and slow migration rate, the number of ejected particles (not shown) continues to increase.

conditions, a large fraction of the planetesimals remain on relatively cool orbits. However, even for fast-migrating, low-mass giant planets, the average eccentricity in the swarm of planetesimals is still increased tenfold during the simulation. Inclinations are similarly increased, but with larger deviations. We also observe retrograde orbits in the simulations with the most massive or slowest migrating planets, but they represent only a few percent of the swarm.

In the simulations with massive planets or slow migration rates, the passage of the giant planet is catastrophic to most of the disk of planetesimals. For a $2 M_{\text {Jup }}$ planet migrating at $10^{-4} \mathrm{AU} \mathrm{yr}^{-1}$, nearly $20 \%$ of the particles are ejected, another $50 \%$ have eccentricity greater than 0.8 , and $5 \%$ are put on nearly Sun-grazing orbits. The case of a $1 M_{\text {Jup }}$ planet migrating at $10^{-5} \mathrm{AU} \mathrm{yr}^{-1}$ is even more destructive. Figure 5 shows the breakdown of final orbits as a function of the planet mass and migration rate. As we describe in $\S 4.3$, much of the original disk will never dynamically cool, and thus cannot play a further role in planet formation. However, note that only about half of the solid material is removed.

The planetesimals are scattered during close encounters with the giant planet. At the giant planet migration rates we used, nearly every planetesimal will undergo such an encounter. To see this, consider the synodic period of a planetesimal and the giant separated by the Hill radius of the giant. The distance the giant migrates inward during this period is (in all our simulations) less than the Hill radius. Therefore, the planetesimal will eventually come within the Hill sphere of the giant planet and be gravitationally scattered. Planetesimals that are pushed into resonances may avoid a close encounter, but will still be excited via the resonance. Planetesimals with inclined orbits can avoid the giant by moving out of the plane. We ran an additional suite of simulations in which we increased the mean inclination of the initial disk of planetesimals. As expected, we found that a puffier disk resulted in more surviving planetesimals.

Edgar \& Artymowicz (2004) presented simulations covering a different range of parameter space from that discussed above. 

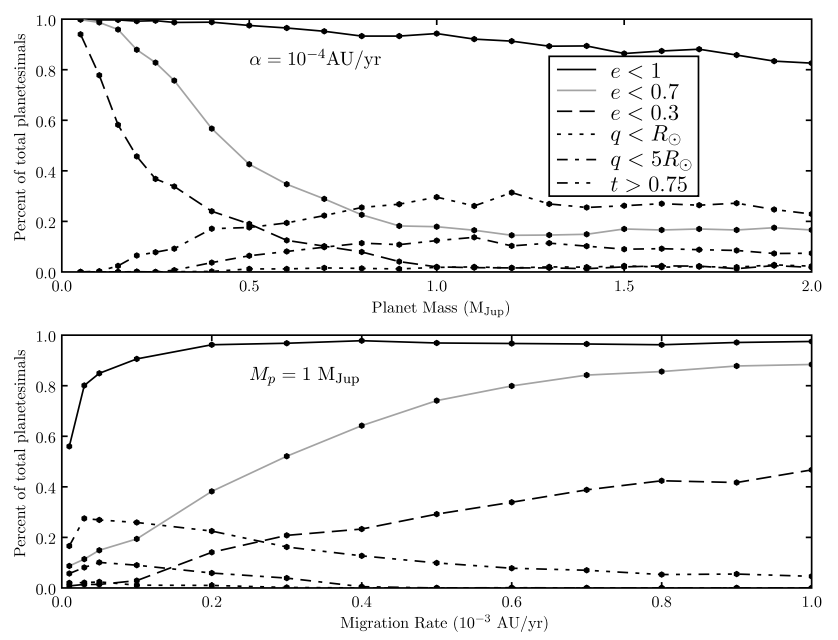

FIG. 5.-Fates of planetesimal orbits at the end of the simulations, as a function of planet mass and migration rate. Shown are the percentage of planetesimals with orbits satisfying different criteria at the end of each simulation, where $e$ is the eccentricity, $q$ the perihelion distance, $R_{\odot}$ the radius of Sun, and $t$ the fraction of the orbital period spent outside one scale height of the disk. The solid line shows bound orbits: more massive migrating giant planets and slower migration rates both lead to more unbound planetesimals. The separation between solid black, solid gray, and dashed lines shows that many planetesimals are put on very higheccentricity orbits. The dotted and dash-dotted lines are planetesimals that will pass very close to their star, either impacting or undergoing significant heating and tidal stress. Finally, as many as $20 \%$ of planetesimals can be put on orbits that are mostly above and below the original disk (double dot-dashed line); these planetesimals will feel minimal drag force from the gas disk. [See the electronic edition of the Journal for a color version of this figure.]

Specifically, they looked at the effect of very rapid migration of $10-200 M_{\oplus}$ planets on a narrow ring of very dynamically cool planetesimals. Our technique is identical, except that it uses a different orbital integrator. We replicated three of their initial conditions, ran the simulations, and measured the final mean and median excitations. For giant planets of masses 10, 100, and $200 M_{\oplus}$ migrating at $0.021 \mathrm{AU} \mathrm{yr}^{-1}$, our results agree to within $20 \%$.

Unlike the giant planets used in our simulations, many extrasolar planets are on eccentric orbits (Schneider 2005). One possible explanation for these orbits is interaction with another planet that either has since been ejected or has not yet been detected (Zakamska \& Tremaine 2004; Ford et al. 2001). In such a case, migration would not be smooth, nor would the planets be constrained to circular orbits, both critical features of our simulations. We expect that such a system would lead to even more excitation than what we have found, as a planet on an eccentric orbit will cross the orbits of more planetesimals per unit time than the same planet on a circular orbit. Simulations of such a scenario are a possible future extension of this work.

\subsection{Cooling the Scattered Disk}

We have shown that a migrating giant planet dynamically excites a cool disk of planetesimals. There are physical interactions that we have not modeled that will act to cool the resultant scattered disk, including gas drag, collisions, and dynamical friction.

We can estimate the eccentricity damping time due to gas drag using the prescription of Adachi et al. (1976). They assume an axisymmetric gas disk with no vertical structure, and derive for planetesimals with small eccentricities $(e \lesssim 0.3)$ an eccentricity decay timescale of

$$
T=\frac{1}{e^{2}} \frac{2 m}{C_{D} \pi r^{2} \rho_{\mathrm{gas}} a \Omega}
$$

where $m, r, a, e$, and $\Omega$ are the mass, radius, semimajor axis, eccentricity, and orbital angular speed of the planetesimal, respectively; $C_{D}$ is the gas drag coefficient (of order unity); and $\rho_{\text {gas }}$ is the gas density at the orbit of the planetesimal. The minimum mass solar nebula model suggests that the gas density is $\rho(r)=$ $1.4 \times 10^{-9}(r / 1 \mathrm{AU})^{-11 / 4} \mathrm{~g} \mathrm{~cm}^{-3}$ (Thommes et al. 2003).

For a $100 \mathrm{~km}$ rocky body in the terrestrial region, the estimated damping timescale is on the order of tens of thousands of years. Compared to the lifetime of the disk, at least a million years, this seems to suggest that an excited swarm of planetesimals will quickly cool to the point where collisional growth is again possible. However, this estimate is valid only for small eccentricities, significantly less than the average eccentricity seen in some of our simulations. More importantly, our simulations show that disks of planetesimals will be significantly puffed up by a migrating giant planet (Fig. 4 shows median inclinations of many tens of degrees). For each planetesimal in the final output of our simulations, we can calculate the percentage of orbital period spent within two scale heights of the disk. The scale height used is $h(r)=0.0472(r / 1 \mathrm{AU})^{5 / 4} \mathrm{AU}$, that of the minimum mass solar nebula model (Thommes et al. 2003). While almost all the planetesimals are on high-eccentricity, highinclination orbits, about half of these orbits are oriented such that they remain in the original plane of the disk. The other half of the swarm spends almost no time in the plane (see the line labeled $t>0.75$ in Fig. 5), and will therefore not be able to cool via gas drag.

Collisions between solid bodies will have a dynamically cooling effect when the velocity dispersion is of the same order as the escape speed of the bodies (de Pater \& Lissauer 2001). The high eccentricities seen in our simulations suggest that any collisions between planetesimals will be at high speed, and hence disruptive, grinding down the bodies and further slowing planet formation.

Another physical interaction capable of cooling the disk is dynamical friction, whereby the eccentricity and inclination of the larger planetesimals in a swarm will be damped by gravitational interaction with the more numerous smaller bodies. Because our simulations used massless planetesimals, we cannot observe this interaction. This effect is important only if there is a size distribution in the swarm of planetesimals.

Gas drag, collisions, and dynamical friction will act to cool a moderately excited disk of planetesimals, such as found in the low-mass, fast-migration rate region of our parameter search. In such cases, only a few percent of the large solid bodies will be lost to planet formation due to the migration event. However, for many other outcomes we observed, a majority of the planetesimals cannot be cooled quickly or efficiently enough to play a further role in terrestrial planet formation. The remaining material will dynamically cool, but very slowly, and may be ground to dust via disruptive collisions. Note that this removal of material, while significant, is not complete, as assumed in some previous studies (Armitage 2003).

\section{CONCLUSIONS}

To investigate survivability of planetesimal disks in the presence of complete giant planet migration, we ran a suite of simulations varying the mass and migration rate of the giant planet. We found that survivability strongly depends on these parameters. If giant planets start migrating at low mass, pre-existing planetesimals interior to the giant may remain on cool orbits after the passage, leaving open the possibility of further growth via accretion. Alternatively, planetesimals can survive even in the presence of very massive planets if migration occurs at the high end of migration rates suggested by previous simulations. There 
remains a large range of parameter space where the presence of an exterior migrating giant planet is catastrophic to about half of any interior dynamically cool planetesimals.

The magnitude of excitation of the swarm that we found is consistent with the simulation in Fogg \& Nelson (2005) without expending computational effort on their more complete physics. In light of this, we recommend the technique used in this paper as a cheap test of survivability, when tighter constraints on planet migration rates eventually become available.

After the passage of a giant planet, a disk of planetesimals must dynamically cool before further growth is possible. The main method for this cooling is via gas drag. We show that gas drag can cool a moderately heated disk in a short amount of time compared to the expected lifetime of the gaseous nebula. However, many of the simulations we ran had final states in which most of the planetesimals were far too dynamically excited to be easily cooled via gas drag, primarily because of the greatly increased average inclination. This population of planetesimals on highly eccentric and inclined orbits may survive for the lifetime of the system. Detecting such objects in our or extrasolar systems would be evidence suggesting a prior migration event.

Many, perhaps all, giant planets will migrate within their solar system during and after their formation. The passage of a migrating planet through a swarm of smaller planetesimals is a transitory event with long-term consequences. The survival of planetesimals in systems where this occurs is strongly dependent on the mass and migration rate of the giant planet. In systems where a giant planet migrates quickly, while still forming, planetesimals will be dynamically excited, but will be able to cool and undergo additional growth following the passage of the giant planet. Because of this, we predict that terrestrial planets will be found exterior to some hot Jupiter systems.
Adachi, I., Hayashi, C., \& Nakazawa, K. 1976, Prog. Theor. Phys., 56, 1756 Armitage, P. J. 2003, ApJ, 582, L47

Chambers, J. E. 2001, Icarus, 152, 205

de Pater, I., \& Lissauer, J. J. 2001, Planetary Sciences (Cambridge: Cambridge Univ. Press)

Edgar, R., \& Artymowicz, P. 2004, MNRAS, 354, 769

Fogg, M. J., \& Nelson, R. P. 2005, A\&A, 441, 791

Ford, E. B., Havlickova, M., \& Rasio, F. A. 2001, Icarus, 150, 303

Jacobsen, S. B., \& Yin, Q. 2003, Lunar Planet Sci. Conf., 34, 1913

Kokubo, E., \& Ida, S. 2002, ApJ, 581, 666

Mandell, A. M., \& Sigurdsson, S. 2003, ApJ, 599, L111

Masset, F. S., \& Papaloizou, J. C. B. 2003, ApJ, 588, 494

Mayer, L., Quinn, T., Wadsley, J., \& Stadel, J. 2004, ApJ, 609, 1045

\section{REFERENCES}

Nelson, A. F., \& Benz, W. 2003, ApJ, 589, 556

Pollack, J. B., Hubickyj, O., Bodenheimer, P., Lissauer, J. J., Podolak, M., \& Greenzweig, Y. 1996, Icarus, 124, 62

Richardson, D. C., Quinn, T., Stadel, J., \& Lake, G. 2000, Icarus, 143, 45

Schneider, J. 2005, The Extrasolar Planets Encyclopaedia (Paris: CNRS), http://www.exoplanet.eu

Stadel, J. G. 2001, Ph.D. thesis, Univ. Washington

Thommes, E. W., Duncan, M. J., \& Levison, H. F. 2003, Icarus, 161, 431

Ward, W. R. 1997, Icarus, 126, 261

Yin, Q., Jacobsen, S. B., Yamashita, K., Blichert-Toft, J., Télouk, P., \& Albarède, F. 2002, Nature, 418, 949

Zakamska, N. L., \& Tremaine, S. 2004, in AIP Conf. Proc. 713, The Search for Other Worlds, ed. S. S. Holt \& D. Deming (Melville: AIP), 257 